\title{
Electron Holography of Dipolar Magnetism in Self-assembled Nanoparticle Chains
}

\author{
Marco Beleggia ${ }^{1}$, Miriam Varón ${ }^{2,5}$, Takeshi Kasama ${ }^{1}$, Rafal E. Dunin-Borkowski ${ }^{3,1}$, Victor F. Puntes ${ }^{2}$, \\ Richard J. Harrison ${ }^{4}$ and Cathrine Frandsen ${ }^{5}$ \\ 1. Center for Electron Nanoscopy, Technical University of Denmark, 2800 Kgs. Lyngby, Denmark \\ 2. Institut Catala' de Nanotecnologia, Campus UAB, 08193 Barcelona, Spain \\ ${ }^{3 .}$ Ernst Ruska-Centre for Microscopy and Spectroscopy with Electrons and Peter Gruenberg Institute, \\ Forschungzentrum Juelich, 52425 Juelich, Germany \\ 4. Department of Earth Sciences, University of Cambridge, Cambridge CB2 3EQ, United Kingdom \\ ${ }^{5 .}$ Department of Physics, Technical University of Denmark, 2800 Kgs. Lyngby, Denmark
}

Nanoparticle magnets are a novel class of materials, in which a lattice of magnetic ions is replaced by a meta-lattice of magnetic nanoparticles. Inter-particle exchange interactions are absent, while dipolar interactions dominate. As a result, nanoparticle magnets behave differently from conventional magnets and their properties may be controlled and tuned by selecting the spacing and compositions of the constituent nanoparticles. We have studied dipolar interactions in self-assembled Co nanoparticle magnets using off-axis electron holography in the transmission electron microscope (TEM). The technique enables the orientation and magnitude of the magnetic moment of each nanoparticle in an array to be determined and correlated with the structural properties of the meta-lattice, including the degree of order of the particles and their size distribution. Our study reveals that dipolar interactions are sufficiently strong to support long-range ferromagnetic order, even when the lattice of nanoparticles is highly disordered. This observation supports the possibility of creating amorphous dipolar magnets, in contrast to the expectation that a disordered dipolar system necessarily implies spin-glass behavior [1].

Chain-like assemblies of $15 \mathrm{~nm} \varepsilon$-Co particles were prepared with an oleic acid coating on a carbon substrate (with no external magnetic field applied). Figure 1a shows TEM bright-field images of representative particle assemblies. For chains that are wider than 1 particle across, the particles are typically assembled into triangular (close-packed) lattices, although square lattice arrangements are also occasionally seen. We used off-axis electron holography to map the projected magnetic fields of several elongated nanoparticle assemblies non-invasively with a nominal spatial resolution of $6.3 \mathrm{~nm}$ (the holographic interference fringe spacing was $2.1 \mathrm{~nm}$ ). Figure $1 \mathrm{~b}$ shows color-coded magnetic induction maps acquired at remanence using electron holography after applying an off-plane field of $2 \mathrm{~T}$ to the specimen. The maps reveal the magnetic moment topography of each chain directly.

In order to quantify dipolar ferromagnetic order, we estimated the magnitude and orientation of the magnetic moment of each individual particle, as shown in Fig. 2a for chain IV. The measurements were correlated with the geometrical arrangements of the particles. For each pair of particles, we measured the spatial separation $r$ between their centers and the angular difference $\Delta \theta$ between their moments. Figure $2 \mathrm{~b}$ shows the distribution $(\mathrm{r}, \Delta \theta)$ for chain IV in Fig. 1, from which magnetic and lattice order parameters were determined. Our results show that short-range magnetic order with small domains dominates the initial states, with the local magnetic order (ferromagnetic or antiferromagnetic) often depending on the particle lattice (triangular or square). In contrast, at remanence after saturation, overall dipolar ferromagnetic order is extremely persistent even in case of a non-triangular lattice. We interpret our results as supporting the existence of amorphous dipolar ferromagnets: i.e., dipolar ferromagnetism in elongated nanoparticle assemblies even in the absence of underlying crystallinity [2]. 


\section{References:}

[1] M Varon et al., Sci. Rep. 3 (2013), p. 1234.

[2] MV thanks the Spanish MICINN for financial support through scholarship BES-2007-17164. CF thanks the Danish Research Council for Independent Research for funding (Steno stipend).

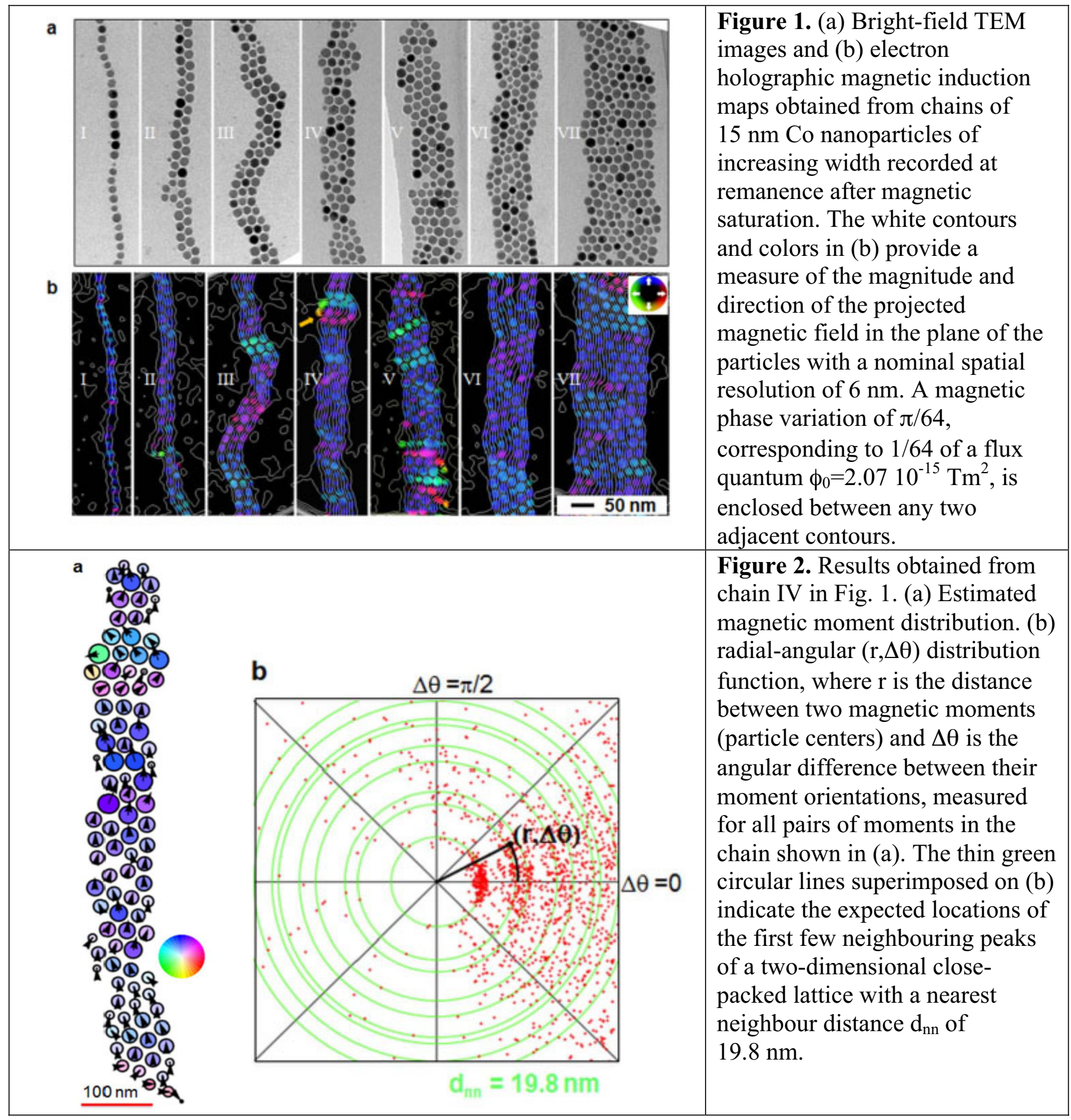

